Cluj-Napoca, Romania; ${ }^{4}$ Pulmonology Hospital “Leon Daniello, Cluj-Napoca, Romania

Background: Interstitial lung disease (ILD) is a common manifestation of connective tissue diseases (CTDs), and is associated with significant morbidity and mortality. Chest high-resolution computed tomography (HRCT) play an important role in the diagnosis of ILD and may provide prognostic information.

Objectives: We aimed to characterize the clinical profile and chest HRCT abnormalities and patterns of patients diagnosed with CTDs and ILD.

Methods: In this retrospective, observational study we included 80 consecutive patients with CTDs and ILD referred to a tertiary rheumatology center between 2015 and 2019. From hospital charts we collected clinical data, immunologic profile, chest HRCT findings. HRCT patterns were defined according to new international recommendations.

Results: Out of 80 patients, $64(80 \%)$ were women, with a mean age of 55 years old. The most common CTD associated with ILD was systemic sclerosis (38.8\%), followed by polymyositis (22.5\%) and rheumatoid arthritis (18.8\%). The majority of patients had dyspnea on exertion $(71.3 \%)$, bibasilar inspiratory crackles were present in $56.3 \%$ patients and $10 \%$ had clubbing fingers. Antinuclear antibodies (ANA) were present in $78.8 \%$ patients, and the most frequently detected autoantibodies against extractable nuclear antigen were anti-Scl 70 (28.8\%), followed by anti-SSA (anti-Ro, 17.5\%), anti-Ro52 (11.3\%) and anti-Jo $(7.5 \%)$. Intravenous cyclophosphamide therapy for $6-12$ months was used in $35 \%$ of patients, while $5 \%$ of patients were treated with mycophenolate mofetil.

The most frequent HRCT abnormalities were reticular abnormalities and ground glass opacity. Non-specific interstitial pneumonia (NSIP) was identified in $46.3 \%$ CTDs patients. A pattern suggestive of usual interstitial pneumonia (UIP) was present in $32.5 \%$ patients, mainly in patients with systemic sclerosis. In $21.3 \%$ patients the HRCT showed reticulo-nodular pattern, micronodules and other abnormalities, not diagnostic for UIP or NSIP pattern.

Conclusion: Nonspecific interstitial pneumonia (NSIP) is the most common HRCT pattern associated with CTDs. Further prospective longitudinal studies are needed in order to determine the clinical and prognostic significance of various HRCT patterns encountered in CTD-associated ILD and for better patient management.

References:

[1] Ohno Y, Koyama H, Yoshikaua T, Seki S. State-of-the-Art Imaging of the Lung for Connective Tissue Disease (CTD). Curr Rheumatol Rep. 2015;17(12):69.

[2] Walsh SLF, Devaraj A, Enghelmeyer JI, Kishi K, Silva RS, Patel N, et al. Role of imaging in progressive-fibrosing interstitial lung diseases. Eur Respir Rev. 2018;27(150)

Disclosure of Interests: None declared

DOI: 10.1136/annrheumdis-2020-eular.3758

\section{AB0606 SYSTEMIC SCLEROSIS - ARE PATIENTS WITH CALCINOSIS DIFFERENT FROM THOSE WHO DO NOT HAVE IT?}

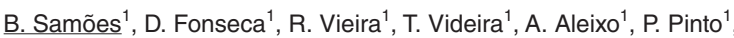
M. Guerra ${ }^{1} .{ }^{1}$ Centro Hospitalar Vila Nova de Gaia, Rheumatology, Vila Nova de Gaia, Portugal

Background: Systemic Sclerosis (SS) is a heterogenous disease with a broad range of organ involvement. Calcinosis is a common problem and although it may affect almost any body tissue, it is typically seen in the limbs. ${ }^{1}$ Its presence relates with higher risk of digital ulcers and infection. ${ }^{2}$ It is still unknown whether patients with calcinosis also have other clinical features that differentiate them from the remaining.

Objectives: To determine the prevalence of calcinosis in a SS cohort and to evaluate if its presence relates with specific clinical features.

Methods: A cross-sectional study was conducted evaluating a cohort of SS patients. Plain radiographs were taken to assess calcinosis at elbows, hands, knees and feet. Clinical data was obtained and analyzed using IBM SPSS Statistics $26 \circledast$.

Results: We included 25 patients, 21 females [ $n=21(84 \%)$ ], median (min, $\max )$ age was $58(27,75)$ years-old. Regarding disease classification, 16 $(64 \%)$ had limited SS, 4 (16\%) had diffuse SS, 3 (12\%) had overlap syndrome and $2(8 \%)$ had early SS. Ten $(40 \%)$ patients had radiological calcinosis in at least one site, seven of which $(70 \%)$ were subclinical. The most affected areas were knees and hands $[n=6(24 \%)]$. Table 1 summarizes the clinical characteristics of patients with and without calcinosis. Limited SS was significantly more prevalent in the calcinosis group $[n=9(90 \%)$ vs. $n=7(46.7 \%), p=0.04]$. All patients had Raynaud phenomenon [ $n=10(100 \%)$ vs. $15(100 \%)]$. Current or past digital ulcers $[n=5(50 \%)$ vs. $n=6(40 \%), p=0.697]$, telangiectasias $[n=9$ $(90 \%)$ vs. $n=11(73.3 \%), p=0.615]$, pulmonary hypertension $[n=2(20 \%)$ vs. $n=1(6.7 \%), p=0.550]$ and esophageal involvement $n=6(60 \%)$ vs. $n=6(40 \%)$, $p=0.428]$ were more frequent in the calcinosis group but with no statistical significance. Although late capillaroscopic pattern was more frequent in the calcinosis group, there was no statistical significance difference $[n=4(40 \%)$ vs. $n=1(6.7 \%), p=0.121]$. Seropositivity for centromere-B antibodies was more frequent in the calcinosis group but with no statistical significance $[n=7(70 \%)$ vs. $n=8(53.3 \%), p=0.678]$.

Table 1. Demographic and clinical data of patients with and without calcinosis.

\begin{tabular}{lccc}
\hline Demographic and clinical data & Calcinosis (n=10) & No calcinosis (n=15) & p-value \\
\hline Female gender, $\mathrm{n}(\%)$ & $9(90)$ & $12(80)$ & 0.626 \\
Age (years), median [min,max] & $68.5[27,75]$ & $52[36,73]$ & 0.129 \\
Cutaneous classification & & & \\
Limited, $\mathrm{n}(\%)$ & $9(90)$ & $7(46.7)$ & 0.04 \\
Diffuse, $\mathrm{n}(\%)$ & $1(10)$ & $3(20)$ & 0.626 \\
Early, $\mathrm{n}(\%)$ & $0(0)$ & $2(13.3)$ & 0.500 \\
Overlap, $\mathrm{n}(\%)$ & $0(0)$ & $3(20)$ & 0.250 \\
Clinical manifestations & & & \\
Current or previous digital ulcers, $\mathrm{n}(\%)$ & $5(50)$ & $6(40)$ & 0.697 \\
Interstitial lung disease, $\mathrm{n}(\%)$ & $2(20)$ & $4(26.7)$ & 1.000 \\
Pulmonary hypertension, $\mathrm{n}(\%)$ & $2(20)$ & $1(6.7)$ & 0.550 \\
Arthritis, $\mathrm{n}(\%)$ & $2(20)$ & $3(20)$ & 1.000 \\
Calcinosis, $\mathrm{n}(\%)$ & $3(30)$ & $0(0)$ & 0.052 \\
Esophageal involvement, $\mathrm{n}(\%)$ & $6(60)$ & $6(40)$ & 0.428 \\
NFC patterns & & & \\
Non specific abnormalities, $\mathrm{n}(\%)$ & $1(10)$ & $3(20)$ & 0.626 \\
Early scleroderma, $\mathrm{n}(\%)$ & $1(10)$ & $1(6.7)$ & 1.000 \\
Active scleroderma, $\mathrm{n}(\%)$ & $3(30)$ & $10(58.8)$ & 0.111 \\
Late scleroderma, $\mathrm{n}(\%)$ & $1(6.7)$ & 0.121 \\
Autoantibodies & $4(40)$ & & \\
Centromere B, $\mathrm{n}(\%)$ & $7(70)$ & $8(53.3)$ & 0.678 \\
Scl-70, $\mathrm{n}(\%)$ & $1(10)$ & $4(26.7)$ & 0.615 \\
\hline
\end{tabular}

Conclusion: The prevalence of calcinosis was similar to that reported in literature $(18-49 \%)$. This study confirmed the association, already found in previous studies, between calcinosis and the limited form of SS and raises attention for the importance of calcinosis radiographic screening since there was a high prev alence of subclinical calcinosis. ${ }^{1}$ Although there were some clinical differences between patients with and without calcinosis, given the small cohort, statistical significance was not obtained. Larger studies are needed to increase statistica power.

References:

[1] Valenzuela A et al. Calcinosis in scleroderma. Curr Opin Rheumatol. 2018 Nov;30(6):554-561.

[2] Bartoli $F$ et al. Calcinosis in systemic sclerosis: subsets, distribution and complications. Rheumatology (Oxford). 2016 Sep;55(9):1610-4

Disclosure of Interests: None declared

DOI: 10.1136/annrheumdis-2020-eular. 1526

\begin{tabular}{|l} 
AB0607 \\
MYOSITIS-RELATED INTERSTITIAL LUNG \\
DISEASES: CLINICAL FEATURES, BIOMARKERS \\
AND AUTOANTIBODIES IN LATINOAMERICAN \\
PATIENTS
\end{tabular}

S. M. Sánchez Romo ${ }^{1}$, G. G. Sánchez Mendieta ${ }^{1}$, M. A. Villarreal-Alarcón ${ }^{1}$, I. D. J. Hernandez-Galarza ${ }^{1}$, D. Á. Galarza-Delgado ${ }^{1}{ }^{1}$ Hospital Universitario Dr. José Eleuterio González UANL, Rheumatology, Monterrey, Mexico

Background: The lung is one of the most common extra-muscular targets in idiopathic inflammatory myopathies (IIM) and interstitial lung disease (ILD) is a prevalent and often devastating manifestation of IIM ${ }^{1}$.

Objectives: To know the frequency of autoantibodies associated with IMM-ILD biomarkers, and their relation with clinical features in patients with IIM.

Methods: Adults with IIM were enrolled in a retrospective way. Demographics, clinical and laboratory features were registered. The determination of antibodies was perfomed by the Inmunoblot technique with Euroinmmun kit. Patients without a myositis antibody panel were excluded. The diagnosis of ILD was based on HRCT. Patients with anti-MDA5 antibodies and with ant Ro-52 antibodies associated with anti-ARS were considered as high risk group, those with anti-ARS, anti-U1-RNP, anti-PM/ Scl and anti-Ku antibodies as moderate risk and those with anti-Mi2, anti SRP and anti TIF1 antibodies as low risk ${ }^{2}$.

Results: Demographics characteristics are shown in table 1. We included 36 patients. Dermatomyositis (DM) was described in $69.4 \%$, polymyositis (PM) in $16.7 \%$ and antysnthetase syndrome (AAS) in $13.9 \%$. Out of the total of our patients, $30.6 \%$ had interstitial lung disease. The most frequent autoantibody was Anti Ro52 in 13 (36.1\%) patients and 44.4\% were in the high risk group. We analized our patients by the presence or absence of ILD and we found that 
anti-MDA5 antibodies were more frequent in IMM-ILD group $(\mathrm{p}=0.006)$. In our IMM-ILD group we compared the autoantibodies with the clinical and serological features and we found that patients with IMM- ILD and anti Ro-52 antibody had more frequency of heliotrope rash $(p=0.045)$ and those with IMM- ILD and anti-Jo1 antibody had a higher level of CK $(p<0.001)$.

Table 1. Demographic characteristics

\begin{tabular}{lc}
\hline Age, mean SD & $40.611+/-16.37$ \\
Women, $\mathbf{n}(\%)$ & $24(66.7 \%)$ \\
Diagnosis & \\
$\quad$ Dermatomiositis & $25(69.4 \%)$ \\
Polimiositis & $6(16.7 \%)$ \\
$\quad$ Síndrome antisintetasa & $5(13.9 \%)$ \\
Time of evolution * & $6.00(2.25-19.00)$ \\
Interstitial lung disease & $11(30.6 \%)$ \\
Serological & \\
CK & 305.00 \\
& $(50.75-1969.250)$ \\
LDH & $460.727+/-384.76$ \\
Risk ILD complicated with IIM & $16(44.4 \%)$ \\
High & $8(22.2 \%)$ \\
Moderate & $12(33.3 \%)$ \\
Low & \\
\hline
\end{tabular}

*months

Table 2. Clinical and serological comparation between IMM groups with and without ILD

\begin{tabular}{lccc}
\hline & IMM with ILD, $\mathbf{n = 1 1}$ & IMM without ILD, $\mathbf{n = 2 5}$ & $\boldsymbol{p}$ \\
\hline Proximal muscle weakness & $10(90.9 \%)$ & $22(88.0 \%)$ & NS \\
Heliotrope Rash & $7(63.6 \%)$ & $10(40.0 \%)$ & NS \\
Mechanic hands & $4(36.4 \%)$ & $4(16.0 \%)$ & NS \\
Anti-Mi-2 & $2(18.2 \%)$ & $8(32.0 \%)$ & NS \\
Anti-Tif1 $y$ & $0(0.0 \%)$ & $6(24.0 \%)$ & NS \\
Anti-MDA5 & $4(36.4 \%)$ & $0(0.0 \%)$ & .006 \\
Anti-NXP2 & $1(9.1 \%)$ & $0(0.0 \%)$ & NS \\
Anti-SAE1 & $0(0.0 \%)$ & $1(4.0 \%)$ & NS \\
Anti-Ku & $0(0.0 \%)$ & $2(8.0 \%)$ & NS \\
Anti-PM/Sc1100 & $0(0.0 \%)$ & $1(4.0 \%)$ & NS \\
Anti-PM/Scl75 & $2(18.2 \%)$ & $0(0.0 \%)$ & NS \\
Anti-Jo1 & $1(9.1 \%)$ & $0(0.0 \%)$ & NS \\
Anti-SRP & $0(0.0 \%)$ & $3(12.0 \%)$ & NS \\
Anti- PL12 & $0(0 \%)$ & $1(4.0 \%)$ & NS \\
Anti-EJ & $1(0.1 \%)$ & $1(4.0 \%)$ & NS \\
Anti- PL7 & $1(9.1 \%)$ & $4(16.0 \%)$ & NS \\
Anti-OJ & $0(0.0 \%)$ & $2(8.0 \%)$ & NS \\
Anti-Ro52 & $5(45.5 \%)$ & $8(32.0 \%)$ & NS \\
& & &
\end{tabular}

Conclusion: Anti-MDA5 antibodies were more frequent in our IMM-ILD group than in IMM without ILD group. Almost half of our patients were in a high risk group, which means they need an early immunosuppressive treatment. In our IMM-ILD patients we found an association between the presence of anti Ro-52 antibody with heliotrope rash and of anti-Jo1 antibody to a higher level of CK.

References:

[1] Saketkoo, L. A., et al. (2010). Interstitial lung disease in idiopathic inflammatory myopathy. Current Rheumatology Reviews, 6(2), 108-119.

[2] H. Yoshifuji, "Biomarkers and Autoantibodies of Interstitial Lung Disease with Idiopathic Inflammatory Myopathies," Clin. Med. Insights Circ. Respir. Pulm. Med., vol. 9s1, pp. 141-147, 2015.

Disclosure of Interests: None declared

DOI: 10.1136/annrheumdis-2020-eular.5098

\section{AB0608 CARDIAC VESSELS CALCIFICATION IN A COHORT OF SYSTEMIC SCLEROSIS PATIENTS: POSSIBLE ROLE IN VASCULOPATHY AND HEART ABNORMALITIES.}

S. Sciacca ${ }^{1}$, C. Rotondo ${ }^{1}$, A. Corrado ${ }^{1}$, L. Giardullo ${ }^{1}$, S. Stefania ${ }^{1}$, A. Altomare ${ }^{1}$, F. P. Cantatore ${ }^{1} .{ }^{1}$ University of Foggia, Rheumatology Unit, Foggia, Italy

Background: Cardiovascular disease is the leading cause of morbidity and mortality worldwide. Myocardial calcifications have been related with cardiovascular diseases (CVD) such as focal wall motion abnormalities and arrhythmias. The impact of vascular calcifications is under investigation in order to define the risk of cardiovascular events. The relationship between cardiac calcification and systemic sclerosis (SSc) has not been investigated.

Objectives: The aim of the study is to evaluate the frequency of different patterns of cardiac calcification in SSc patients, and to correlate them to other CVD risk factors.
Methods: We analyzed thoracic-CT scanners of 35 SSc patients (88\% female, aged 47,8 ys $\pm 12,9$, disease duration 12,8 ys \pm 9 ) to determine the location and extension of vascular and cardiac calcification. All recruited patients fulfilled the 2013 ACR-EULAR classification criteria for SSc. No one patients had renal failure, cardiomyopathy, myocarditis, history of cardiac surgery or radiotherapy. Results: We found myocardial vessels calcifications (MCv) in 37\% SSc patients, aortic wall calcifications (ACW)in $60 \%$ SSc patients, cardiac valve calcifications (VC) in $28 \%$ SSc patient and heart wall calcifications (HCW) in $20 \%$.

The SSc patients with almost one calcification had older age $(65 \pm 9,8$ ys vs $50 \pm 8,8$ ys; $p=0,0001)$ and higher values of circulating NTproBNP $(336,9 \pm 351,9$ vs $144,2 \pm 107,8 ; p=0,04)$ compared to those without

In particular, the SSc patients with MCv had and uric acid $(5,3 \pm 1,5$ vs $4,1 \pm 1,3$; $\mathrm{p}=0,05)$, higher rate of $\mathrm{PAH}(25 \%$ vs $0 \% ; \mathrm{p}=0,037)$, arrhythmia $(38,5 \%$ vs $9 \%$; $\mathrm{p}=0,036)$ and higher prevalence of CENP-B antibodies $(46 \%$ vs $4 \% ; p=0,01)$ compared to patients without MCv.

Patients with $\mathrm{HCw}$ had lower $\mathrm{C}$ reactive protein $(0,16 \pm 0,10$ vs $0,7 \pm 0,7 ; p=0,008)$ compared to those without HCw. No differences in the rate of heart and vascula complications of SSc were observed.

The SSc patients with ACw had higher frequency of arrhythmia (33\% vs $0 \%$ $p=0,016)$ and longer disease duration $(15,5$ y $\pm 9,9$ vs $8,8 \pm 5,8 ; p=0,03)$.

The SSc patients with VC had higher rate of $\mathrm{PAH}(33 \% \mathrm{vs} 0 \% ; \mathrm{p}=0,003)$ and uric acid $(6 \pm 0,5 v s 3,8 \pm 1,2 p=0,0001)$.

Regression analysis excluded any association with gender, BMI, systemic arterial hypertension, steroid therapy, hypovitaminosis D or smoke habit. No cardiovascular event was recorded in one year of observation.

Conclusion: All patterns of calcifications may be related mostly with the older age. Myocardial vessels calcifications have been found in a high percentage of SSc patients and in particular in those with PAH and positive for anti CENP-B. Furthermore, myocardial vessels calcifications could be associated to the higher occurrence of arrhythmia. More studied are needed to assess the importance of vascular calcification as a part of the vascular involvement in SSc.

References:

[1] John W. Nance Jr. MD. Myocardial calcifications: Pathophysiology, etiologies, differential diagnoses, and imaging findings. Journal of Cardiovascular Computed Tomography 9 (2015) 58 e 67.

[2] Pagkopoulou E, Poutakidou M. Cardiovascular risk in systemic sclerosis: Micro- and Macro-vascular involvement. Indian J Rheumatol 2017;12, Suppl S1:211-7

[3] Plastiras SC, Toumanidis ST. Systemic sclerosis: the heart of the matter. Hellenic J Cardiol. 2012;53(4):287-300.

Disclosure of Interests: None declared

DOI: 10.1136/annrheumdis-2020-eular.5746

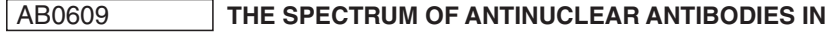 PATIENTS WITH SYSTEMIC SCLEROSIS POSITIVE FOR ANTI-U1RNP}

R. Shayakhmetova ${ }^{1}$, L. P. Ananyeva ${ }^{1}$, O. Koneva ${ }^{1}$, M. Starovoytova ${ }^{1}$,

O. Ovsyannikova', O. Desinova', L. Garzanova ${ }^{1} .{ }^{1}$ V.A. Nasonova Research

Institute of Rheumatology, Moscow, Russian Federation

Background: Patients with systemic sclerosis positive for anti-U1RNP have special clinical picture and disease progression. The autoimmune profile in this group is poorly understood.

Objectives: The purpose of our work was to study the level of major autoantibodies in patients with systemic sclerosis positive for anti-U1RNP.

Methods: The study included 80 patients $(71$ women and 9 men, mean age $44,5 \pm 14$ years) positive for antibodies to RNP and meeting the criteria of the systemic sclerosis (ACR/EULAR 2013). Patients were examined for autoantibodies: RF, ACCP, ACA, anti-Scl70, anti-RNAP-III, anti-Ro, anti-La, anti-dsDNA, anti-Sm, ACL, anti-Jo1. 44 patients were examined in dynamics in 24 months.

Results: In the study group the clinical picture was dominated by inflammatory musculoskeletal lesions (synovitis and myopathy), skin manifestations were poorly expressed. Interstitial lung disease was detected in $68 \%$ of cases. Overlaps (34\%) with other rheumatic diseases (rheumatoid arthritis, systemic lupus erythematosus) and combination with Sjogren's syndrome (32.5\%) were frequently noted. Other antibodies were often detected: commonly - RF (31\%), anti-Ro (38\%), anti-dsDNA (42\%), rarely - anti-Sm (11\%), ACCP (8\%), anti-La (8\%), ACA (6\%), anti-Scl70 (6\%), AKL (2\%). Anti-Jo1 and anti-RNAP-III were not detected at all. In patients with systemic sclerosis highly-positive for antiU1RNP (more than 2 upper normal limits) RF, anti-Ro, anti-dsDNA were significantly more common in comparison with low-positive $(\mathrm{p}=0.00)$. In dynamics $80 \%$ of patients maintained anti-U1RNP, while other autoantibodies were detected with the same frequency. In patients with initially low titer of anti-U1RNP, their disappearance was noted. 